\title{
Instabilidade de pontos de equilíbrio de alguns sistemas lagrangeanos
}

\author{
Ricardo dos Santos Freire Júnior
}

TESE APRESENTADA

$\mathrm{AO}$

Instituto De Matemática e Estatística

DA

Universidade DE SÃo PAUlO

PARA

OBTENÇÃO DO TÍTULO

$\mathrm{DE}$

Doutor EM CIÊNCIAS

Área de Concentração: Matemática Aplicada
Orientador: Prof. Dr. Manuel Valentim de Pera Garcia

Durante o desenvolvimento deste trabalho o autor recebeu auxílio financeiro da CAPES

São Paulo, agosto de 2007 


\section{Instabilidade de pontos de equilíbrio de alguns sistemas lagrangeanos}

Este exemplar corresponde à redação final da tese devidamente corrigida e defendida por Ricardo dos Santos Freire Júnior e aprovada pela comissão julgadora.

Banca examinadora:

- Prof. Dr. Manuel Valentim de Pera Garcia (orientador) - IME-USP

- Prof. Dr. Fábio Armando Tal - IME-USP

- Prof. Dr. Marco Antonio Teixeira - UNICAMP

- Prof. Dr. Orlando Francisco Lopes - UNICAMP

- Prof. Dr. Marcelo Amorim Savi - UFRJ 
A Juliane Karine Ishida 


\section{Agradecimentos}

Em primeiro lugar, agradeço ao meu orientador Manuel Valentim de Pera Garcia. E acredito que eu possa fazer isso com o diferencial de agradecer não só por todos esses anos em que aprendi muito e, certamente, em que aprendi o que sei de Matemática hoje, mas também pela grande amizade que fizemos e apoio que sempre esteve disponível, e não foram poucas ocasiões.

Devo também um agradecimento a muitos professores que participaram, uns mais direta outros mais indiretamente, da minha formação até hoje e dos resultados no presente trabalho. Dentre eles, certamente devo destacar a Sônia Regina Leite Garcia, o professor Ângelo Barone Netto e Fábio Armando Tal.

Do ponto de vista pessoal, muitas pessoas me ajudaram consideravelmente a sobreviver ao longo desses últimos anos e possibilitaram que eu me dedicasse a este trabalho. Nesse sentido agradeço muito a Juliane Karine Ishida, a quem dedico também este trabalho, ao meu grande amigo Bruno Salvatore Drago, a meus pais, irmãos e aos meus tios.

Também quero agradecer de forma geral à turma 9 do Curso de Ciências Moleculares, onde toda minha empolgação com esta vida acadêmica começou e não seria o mesmo sem o convívio com eles.

Finalmente, agradeço a todos que ajudaram na revisão deste texto, em particular à professora Sônia, que muito contribuiu para aparar tantas arestas - em particular pela versão simplificada da prova do teorema central deste trabalho. 
Sou brasileiro e não desisto nunca!

Governo Federal 


\section{Resumo}

Neste trabalho, estudamos algumas inversões parciais do teorema de Dirichlet Lagrange, essencialmente estendendo os resultados em dois graus de liberdade de Garcia e Tal (2003) para algumas situações em $\mathbb{R}^{2 n}$. Mais precisamente, um dos objetivos é mostrar, no contexto da mecânica lagrangeana, que se há um split da energia potencial em uma parte no plano cujo jato $k$ mostra que ela não tem mínimo no ponto de equilíbrio

e existe o jato $k-1$ do seu gradiente, e a outra em $\mathbb{R}^{n-2}$ que tenha mínimo no ponto de equilíbrio, este é instável.

A instabilidade do ponto de equilíbrio em estudo é provada mostrando a existência de uma trajetória assintótica ao mesmo. Para isso, apresentamos um resultado inicial para lagrangeanos com uma forma bem específica e, a seguir, mostramos que a classe de lagrangeanos que descrevemos acima pode ser levada a esta forma, através de uma adequada mudança de coordenadas espaciais.

Além disso, consideramos a extensão desses resultados a sistemas com forças giroscópicas.

Palavras-chave: estabilidade de Liapunov, teorema de Dirichlet-Lagrange, sistemas lagrangeanos. 


\section{Abstract}

In this work, we study some partial inversions of the Lagrange-Dirichlet theorem, extending the results in two degrees of freedom of (GARCIA; TAL, 2003) for some other situations in $\mathbb{R}^{2 n}$. More precisely, one of our objectives is to show, in the context of lagrangian mechanics, that if there is a splitting of the potential energy in one part in the plane which its $k$-jet shows that it does not have a minimum in the equilibrium and there exists the $(k-1)$-jet of its gradient, and the other part in $\mathbb{R}^{n-2}$ has a minimum in the equilibrium, then the equilibrium point is unstable.

Instability of the equilibrium point is shown by proving the existence of an assymptotic trajectory to it. For this purpose, first it is proven a result for lagrangians with a specific form and, next, we show that the class of lagrangians we are interested in can be transformed into this specific form by a subtle change of spatial coordinates.

Finally, we consider the extension of this results to systems with gyroscopic forces.

Keywords: Liapunov stability, Lagrange-Dirichlet theorem, lagrangian systems. 


\section{Sumário}

1 Introdução 1

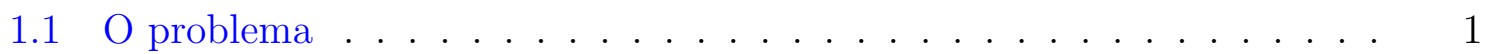

1.2 Hipóteses e notações . . . . . . . . . . . . . . . . . . . . 7

2 Lemas e estimativas iniciais $\quad 12$

3 Teorema de instabilidade $\quad 20$

4 O splitting com o plano 23

5 Sistemas com forças giroscópicas $\quad 28$

6 Considerações diversas $\quad 31$

$6.1 U$ pode ser negativa . . . . . . . . . . . . . . . . . . 32

6.1.1 U e $\pi_{2}$ como funções de duas variáveis . . . . . . . . . . . . . . 33

6.1.2 As condições sobre $U \ldots$. . . . . . . . . . . . . . . . 35

6.2 Alguns exemplos ......................... 37

7 Conclusão $\quad 38$

Referências Bibliográficas $\quad 40$ 


\section{Capítulo 1}

\section{Introdução}

\subsection{O problema}

Consideramos o estudo da instabilidade de Liapunov de pontos de equilíbrio de sistemas lagrangeanos conservativos em $\mathbb{R}^{2 n}$, de lagrangeanos $\mathcal{L}(q, \dot{q})=T(q, \dot{q})-\pi(q)$, sendo $\pi$ a energia potencial e $T$ a energia cinética.

As equações de Lagrange para um sistema desses são

$$
\frac{d}{d t} \frac{\partial \mathcal{L}}{\partial \dot{q}}-\frac{\partial \mathcal{L}}{\partial q}=0
$$

e seus pontos de equilíbrio são da forma $\left(q_{0}, 0\right)$ tais que $\frac{\partial \pi}{\partial q}\left(q_{0}\right)=0$.

Nesse contexto, Lagrange enunciou em 1788 um teorema, provado em 1846 por Dirichlet, o qual diz que se um ponto de equilíbrio é um mínimo estrito local para $\pi$, então este ponto é estável no sentido de Liapunov, sendo este o chamado teorema de Dirichlet-Lagrange.

Por algum tempo, tentou-se mostrar que valia a recíproca do teorema de DirichletLagrange, isto é, se $q_{0}$ é um ponto de equilíbrio do sistema lagrangeano e não é um 
mínimo estrito local de $\pi$, então este seria instável. Em 1904 Painlevé deu um contraexemplo a esta recíproca já em um grau de liberdade. Para isso, considerou o sistema com energia cinética $T(\dot{q})=\frac{\dot{q}^{2}}{2}$ e energia potencial $\pi(q)=e^{-\frac{1}{q^{2}}} \sin \frac{1}{q}$, se $q \neq 0$ e $\pi(0)=0$. Claramente, a origem não é mínimo nem máximo local de $\pi$, mas Painlevé mostrou que $(0,0)$ é um equilíbrio estável das equações de Lagrange.

Desde então, o problema de se estudar a estabilidade de um ponto de equilíbrio que não seja mínimo estrito local para a energia potencial, usualmente dando condições suficientes para a instabilidade do ponto de equilíbrio em estudo, tem sido conhecido como o problema da inversão do teorema de Dirichlet-Lagrange.

Diversos resultados interessantes surgem nesse contexto. Em particular, para um grau de liberdade - e só para este caso, veja Rouche, Habets e Laloy (1977) - o problema está completamente resolvido, no sentido em que existe uma condição necessária e suficiente para a estabilidade do ponto de equilíbrio que é relativamente simples de se verificar.

Em Laloy (1976), um exemplo mais interessante que o de Painlevé é apresentado, onde existe uma semi-reta saindo da origem na qual a energia potencial é negativa, e ainda assim, o ponto de equilíbrio $(0,0)$ é estável. Para isso, considerou a energia cinética $T\left(q_{1}, q_{2}, \dot{q}_{1}, \dot{q}_{2}\right)=\frac{\dot{q}_{1}^{2}+\dot{q}_{2}^{2}}{2}$ e a energia potencial $\pi\left(q_{1}, q_{2}\right)=e^{-\frac{1}{q_{1}^{2}}} \cos \frac{1}{q_{1}}-$ $e^{-\frac{1}{q_{2}^{2}}}\left(\cos \frac{1}{q_{2}}+q_{2}^{2}\right)$. Este sistema é separável, e de forma análoga ao que se prova no exemplo de Painlevé, mostra-se que ele é estável estudando cada uma das suas partes. Entretanto, mostra que mesmo com uma curva que tende à origem em que a energia potencial é estritamente negativa, não se garante a instabilidade da mesma.

Dentro do problema da inversão do teorema de Dirichlet-Lagrange, uma conjectura formulada pelo próprio Liapunov e refeita por Barone Netto com o instrumental introduzido em Barone Netto (1984), serve de guia para o estudo deste problema. 
Conjectura (Liapunov e Barone Netto). Sendo a origem um equilíbrio do sistema lagrangeano, se o jato $k$ de $\pi$ mostra que a energia potencial não tem mínimo na origem, então a esta é um ponto de equilíbrio instável.

Para as definições de jato $k$ e seu uso para garantir que uma função não tenha mínimo, veja a seção seguinte deste capítulo. Sem perda de generalidade, supomos a partir de agora que o ponto de equilíbrio em estudo está localizado na origem.

Esta conjectura, se verdadeira, seria o melhor resultado possível dentro do conjunto das funções que possuem jato de ordem $k$, pois caso o jato $k$ da energia potencial não garanta que esta não tem mínimo na origem, então existe uma função $g$ que tem mínimo estrito na origem e é tal que $j^{k} g=j^{k} \pi$, de onde a origem é estável pelo teorema de Dirichlet-Lagrange.

Para dois graus de liberdade, em Garcia e Tal (2003) esta conjectura é demonstrada com a hipótese adicional de que exista o jato de ordem $k-1$ do gradiente da energia potencial, que é essencialmente uma hipótese técnica.

Diversos outros resultados relevantes podem ser enunciados no contexto desta conjectura. Por exemplo, se o primeiro jato que mostra que a origem não é um mínimo é homogêneo, em Moauro e Negrini (1989) e em Taliaferro (1990) prova-se que, com algumas hipóteses sobre a regularidade do lagrangeano, a origem é instável. O resultado segue demonstrando-se a existência de uma trajetória assintótica à origem, estratégia que é comum a quase todos os resultados recentes que citamos aqui.

Estendendo esse resultado, em Maffei, Moauro e Negrini (1991) o teorema seguinte é provado.

Teorema (Maffei, Moauro e Negrini). Considere o sistema lagrangeano dado pelas equações $(1.1)$ em $\mathbb{R}^{2(m+n)}$, com $q=(u, v), \dot{q}=(\dot{u}, \dot{v})$ e $\mathcal{L}=T-\pi$. Admita que existam 
um natural $k \geq 3$ e reais $\omega_{1}, \ldots, \omega_{m}$ tais que:

1. $\pi(u, v)=\frac{1}{2}\langle u, l(u, v) u\rangle+\pi_{[k]}(u, v)+R(u, v)$, onde $l(u, v)$ é uma matriz $m \times$ $m$ tal que $l(0,0)=\operatorname{diag}\left(\omega_{1}^{2}, \ldots, \omega_{m}^{2}\right), \pi_{[k]}$ é homogêneo de grau $k, R(u, v)=$ $O\left(\|(u, v)\|^{k+1}\right)$ e $\min \left\{\pi_{[k]}(0, v):\|v\|=1\right\}=-1$;

2. $\mathcal{L}$ é de classe $\mathcal{C}^{k+3+\left\lfloor\frac{k-3}{2}\right\rfloor}$.

Então existe uma trajetória $\phi(t)$ tal que $(\phi(t), \dot{\phi}(t)) \rightarrow(0,0)$ com $t \rightarrow-\infty$.

Este resultado, em particular, demonstra a conjectura sob a hipótese de que existe um split de $\mathbb{R}^{n+m}=\mathbb{R}^{n} \oplus \mathbb{R}^{m}$ onde temos que o jato $k$ da energia potencial $\pi$ é $\pi_{2}\left(q_{1}, \ldots, q_{n}\right)+\pi_{k}\left(q_{n+1}, \ldots, q_{m}\right)$, tal que $\pi_{2}$ é uma forma quadrática positiva definida em $\left(q_{1}, \ldots, q_{n}\right)$ e $\pi_{k}$ é um polinômio homogêneo de grau $k$ que mostra que a origem não é um mínimo para a energia potencial; e que $\mathcal{L}$ é de classe $\mathcal{C}^{k+3+\left\lfloor\frac{k-3}{2}\right\rfloor}$.

Finalmente, um resultado que seria uma inversão "completa" (a menos do caso de mínimo não estrito) quando o lagrangeano é analítico foi apresentado em Palamodov (1995), onde se o lagrangeano é analítico e a energia potencial não tem mínimo (nem mesmo brando) na origem, então esta é um equilíbrio instável. Neste último trabalho, apenas a instabilidade é provada e não é assegurada a existência de órbitas assintóticas à origem, ao contrário dos demais trabalhos recentes citados. Graças aos resultados em Barone Netto, Gorni e Zampieri (1996), temos que se $\pi$ é analítica e não tem mínimo na origem, existe $k$ tal que $j^{k} \pi$ mostra isso. Desta forma, uma resposta positiva à conjectura de Liapunov e Barone Netto, é uma generalização também deste resultado ou, mais precisamente, o caso em que o lagrangeano é analítico e a energia potencial não tem mínimo na origem é um caso particular contido na conjectura apresentada.

Neste trabalho, aumentamos a classe de jatos que garantem a instabilidade do ponto de equilíbrio. Para isso, provamos em particular o resultado seguinte. 
Corolário 1. Considere o sistema lagrangeano dado pelas equações (1.1) e admita que $\mathcal{L}=T-\pi$ é tal que 0 é um ponto de equilíbrio, que existe um natural $k \geq 2$ tal que o jato $k$ de $\pi$ mostra que a origem não é um mínimo para $\pi$, que $\mathcal{L}$ é de classe $\mathcal{C}^{k+\left\lfloor\frac{k-3}{2}\right\rfloor+3}$, que o posto de $j^{2} \pi(0)$ é pelo menos $n-2$ e que $T$ é uma forma quadrática positiva definida em $\dot{q}$ para todo $q$. Então existe uma trajetória $\phi(t)$ tal que $(\phi(t), \dot{\phi}(t)) \rightarrow(0,0)$ com $t \rightarrow-\infty$.

Este resultado é uma extensão de Garcia e Tal (2003) para o caso em que $j^{2} \pi(0)$ é uma forma quadrática positiva de posto $n-2$ e, desta forma, fica provado também o caso em que o lagrangeano é analítico e a hessiana da energia potencial tem no máximo 2 autovalores nulos.

Para obter essa extensão, provamos inicialmente um resultado técnico (o teorema 1) que garante a instabilidade de um ponto de equilíbrio para um sistema com várias hipóteses fortes, principalmente sobre a forma da energia potencial. De fato, deve-se notar que este é o principal resultado do trabalho pois, através de uma mudança de coordenadas, inclui o caso desejado e diversos outros casos eventualmente interessantes. Em seguida, mostramos que a classe de sistemas do corolário 1 satisfaz essas hipóteses após mudanças de coordenadas apropriadas.

Nossas técnicas são baseadas nas de Garcia e Tal (2003), onde uma função auxiliar $V$ adequada é escolhida para mostrar algumas propriedades do campo no espaço de fase. A demonstração do resultado em Garcia e Tal (2003) é feita com estimativas muito precisas sobre $\dot{V}$ que possuem pouco espaço para melhorias. Em nossa demonstração, perturbamos a função auxiliar $V$ no nível de energia nula de tal forma que conseguimos, assim, simplificar a demonstração original e obter estimativas análogas para a função perturbada.

Ainda, como um problema relacionado, consideramos sistemas lagrangeanos sob a 
ação de forças giroscópicas, conhecido como o problema de Routh. As equações de Lagrange para um sistema desses são

$$
\frac{d}{d t} \frac{\partial \mathcal{L}}{\partial \dot{q}}-\frac{\partial \mathcal{L}}{\partial q}=Q
$$

onde $Q=Q(q) \dot{q}$ é linear em $\dot{q}$ e tal que $\langle Q(q) \dot{q}, \dot{q}\rangle=0$. Os pontos de equilíbrio são da forma $\left(q_{0}, 0\right)$ onde $\frac{\partial \pi}{\partial q}\left(q_{0}\right)=0$, exatamente como no caso conservativo.

Estabilização giroscópica é um fenômeno conhecido, veja por exemplo Rouche, Habets e Laloy (1977), que mostra a importância de hipóteses adicionais na força giroscópica e, também, que algumas técnicas usadas na demonstração do teorema de Dirichlet-Lagrange podem não funcionar neste contexto. Em particular, muitas vezes utiliza-se da reversibilidade dos sistemas lagrangeanos conservativos para, mais comodamente, provar-se a existência de uma trajetória assintótica à origem com $t \rightarrow+\infty$ e isso garantiria a existência de uma trajetória assintótica à origem com $t \rightarrow-\infty$. Na presença de forças giroscópicas, não podemos usar o argumento de reversibilidade e, por isso, em nossas demonstrações, mostramos diretamente a existência de trajetórias assintóticas à origem com $t \rightarrow-\infty$.

Em Moauro e Negrini (1989), mostra-se que o método usado para tratar o caso em que o jato $k$ de $\pi$ que mostra que a origem não é um mínimo para $\pi$ é homogêneo funciona na presença de forças giroscópicas, sob as hipóteses adicionais de que exista

um inteiro $s$ tal que $s \geq \frac{k+2}{2}$ e $j^{s} Q$ é o primeiro jato não nulo de $Q$. Este resultado é uma extensão do caso analítico em Furta (1986).

Demonstramos aqui que, sob hipóteses sutilmente mais fracas nas forças giroscópicas, que nossos resultados para o caso conservativo provados nesta tese continuam válidos na presença de forças giroscópicas. 
Não esperamos aqui apresentar todos os resultados e contextos em que os problemas já foram estudados, pois a lista é extensa e o leitor interessado poderá encontrar como excelentes pontos de partida o capítulo 3 de Rouche, Habets e Laloy (1977) e uma lista de resultados relevantes é apresentada na introdução de Moauro e Negrini (1989). Nestes trabalhos, encontram-se também algumas discussões e referências para problemas correlacionados, como o de sistemas lagrangeanos com a presença de forças giroscópicas citado.

Este texto está organizado da seguinte maneira: na seção seguinte, damos as definições e notações que usaremos ao longo do texto. No capítulo 2 provamos alguns lemas que serão úteis para provarmos o teorema principal, o que será feito no capítulo 3. No capítulo 4 mostramos então que este resultado estende os de Garcia e Tal (2003), como gostaríamos. No capítulo 5, consideramos a extensão natural dos mesmos resultados para o caso de sistemas com forças giroscópicas. Finalmente, no capítulo 6 fazemos considerações diversas que não julgamos apropriadas nas demais partes do texto sobre os resultados até então e possíveis extensões e concluímos o texto com os comentários finais no capítulo 7.

\subsection{Hipóteses e notações}

As definições e notações inseridas nesta seção serão usadas ao longo do texto. Embora procuremos citar sempre da forma mais precisa a que nos referimos, o contexto muitas vezes deixará suficientemente claro o entendimento.

Precisamos de duas definições básicas sobre $k$-decidibilidade introduzidas por Barone Netto e apresentadas, respectivamente, em Barone Netto (1984) e Garcia e Tal (2003), que usaremos ao longo do trabalho. Trata-se da noção de jato punctual de ordem $k$ de uma função $f$ e de quando este jato mostra que a função não tem mínimo, 
que detalhamos a seguir.

Observamos que a norma utilizada nesta e demais definições é, de fato, irrelevante. Assim, sem perda de generalidade, estaremos pensando sempre que $\|\cdot\|=\|\cdot\|_{2}$, fora menção em contrário.

Definição. Seja $\Omega \subset \mathbb{R}^{n}$ um aberto contendo a origem. Uma função $f: \Omega \rightarrow \mathbb{R}$ tem jato punctual de ordem $k$ na origem, com $k$ natural, se existe um polinômio $P: \mathbb{R}^{n} \rightarrow \mathbb{R}$ de grau menor ou igual a $k$ tal que $\lim _{x \rightarrow 0} \frac{f(x)-P(x)}{\|x\|^{k}}=0$. Neste caso, denotamos o polinômio $P$ por $j^{k} f$.

Em geral, ao longo deste texto, iremos sempre cometer o abuso e chamar simplesmente de jato $k$ o jato punctual de ordem $k$, sempre na origem.

Uma conseqüência simples da definição acima é que se existe o jato $k$ de $f$, este é único, de onde se $f$ tem polinômio de Taylor de ordem $k$, este é o seu jato $k$.

Definição. Diremos que $j^{k} f$ mostra que $f$ não tem mínimo na origem se $f$ é uma função que tem jato punctual de ordem $k$ na origem e, para toda função $g$ tal que $j^{k} f=j^{k} g$ temos que g não tem mínimo (nem estritamente fraco) na origem.

Para podermos especificar o sistema inicial em que trabalharemos, precisamos da definição seguinte.

Definição. Dados reais $\alpha>0$ e $\beta>1$, e uma função $P: \mathbb{R}^{m} \rightarrow \mathbb{R}$, diremos que $P$ satisfaz a propriedade- $(\alpha, \beta)$ se existem reais $\delta \in\left(0, \frac{\alpha}{4}\right)$ e $\sigma>0$ tais que há uma componente conexa $C$ de

$$
\Lambda=\left\{\left(q_{1}, \ldots, q_{m}\right): 0<q_{m}<\sigma, P\left(q_{1}, \ldots, q_{m}\right) \leq \delta q_{m}^{\beta}\right\}
$$


que contém $\left\{\left(0, \ldots, 0, q_{m}\right), 0<q_{m}<\sigma\right\}$, na qual vale a desigualdade

$$
q_{m} \frac{\partial P}{\partial q_{m}}\left(q_{1}, \ldots, q_{m}\right) \leq(k-1) \delta q_{m}^{\beta},
$$

$\operatorname{com} k=\lceil\beta\rceil$.

Esta definição refere-se a funções sobre as quais temos alguma estimativa de sua derivada em uma direção especial, no caso a da sua última coordenada, e será muito importante na determinação do sinal da derivada da função auxiliar que utilizamos nos próximos capítulos, pois ela será escolhida de forma que possamos nos preocupar com a análise dos termos do gradiente da energia potencial apenas nesta direção.

Com isto, podemos definir a classe de lagrangeanos com que começaremos nosso estudo, que são os que se encontram em uma forma particular, dada principalmente pelo splitting da energia potencial e exigências menores sobre a diferenciabilidade do lagrangeano, que são relevantes para que as mudanças de coordenadas feitas no caso do splitting com o plano nos levem a um sistema com estas hipóteses satisfeitas.

Definição. Diremos que o lagrangeano $\mathcal{L}(q, \dot{q})=T(q, \dot{q})-\pi(q)$ satisfaz as hipóteses $\mathcal{H}_{0}$ se valerem:

1. $\mathcal{L}$ está definido para q pertencente a $\Omega \cap\left\{q: q_{n}>0\right\} \cup\{0\}$, para alguma vizinhança aberta $\Omega$ de 0 em $\mathbb{R}^{n}$, e $\dot{q}$ pertencente $a \mathbb{R}^{n}$;

2. $T$ é uma forma quadrática definida positiva em $\dot{q}$ para todo $q$ e sendo $T(q, \dot{q})=$ $\frac{1}{2} \sum_{l, s=1}^{n} g_{l s}(q) \dot{q}_{l} \dot{q}_{s}$, temos que a matriz $\left[g_{l s}(q)\right]$ é simétrica para todo q e existem reais $\mu_{1}>0$ e $\mu_{2}>-1$ tais que $\left[g_{l s}(q)\right]=I+h(q)$, onde $\|h(q)\|=o\left(\|q\|^{\mu_{1}}\right) e$ $\left\|h^{\prime}(q)\right\|=o\left(\|q\|^{\mu_{2}}\right) ;$

3. $\pi$ é de classe $\mathcal{C}^{2}$ para $q_{n}>0$ e contínua em $\Omega \cap\left\{q: q_{n}>0\right\} \cup\{0\}, \pi(0)=0$ e 
existem um natural $N$ e reais $\alpha>0$ e $\beta>1$ tais que

$$
\pi(q)=U\left(q_{1}, \ldots, q_{N}\right)+\pi_{2}\left(q_{N+1}, \ldots, q_{n}\right),
$$

onde:

(a) $U\left(q_{1}, \ldots, q_{N}\right) \geq 0$ para todo $\left(q_{1}, \ldots, q_{N}\right)$;

(b) $\pi_{2}\left(q_{N+1}, \ldots, q_{n}\right)=-\alpha q_{n}^{\beta}+P\left(q_{N+1}, \ldots, q_{n}\right)+R\left(q_{N+1}, \ldots, q_{n}\right)$, sendo

i. $P\left(q_{N+1}, \ldots, q_{n}\right)$ satisfaz a propriedade- $(\alpha, \beta)$ em $\mathbb{R}^{n-N}$;

ii. $P\left(0, \ldots, 0, q_{n}\right)=0$ e $P\left(q_{N+1}, \ldots, q_{n}\right) \geq 0$ para todo $\left(q_{N+1}, \ldots, q_{n}\right) \in C$, onde $C$ é dado pela propriedade- $(\alpha, \beta)$ de $P$;

iii. $R\left(q_{N+1}, \ldots, q_{n}\right)=o\left(\left\|\left(q_{N+1}, \ldots, q_{n}\right)\right\|^{\beta}\right)$;

iv. $\frac{\partial R}{\partial q_{j}}=o\left(\left\|\left(q_{N+1}, \ldots, q_{n}\right)\right\|^{\beta-1}\right)$ para $j=N+1, \ldots, n$.

Ainda, para o capítulo em que finalmente se generaliza os resultados de Garcia e Tal (2003), a seguinte definição é relevante. Nela enunciamos formalmente todos os requisitos que são supostos na conjectura formulada por Liapunov e Barone Netto, e assim especifica em última análise a maior classe de sistemas lagrangeanos que gostaríamos de mostrar a instabilidade. Neste trabalho, mostramos no teorema 2 e corolário 1 que uma subclasse desta é de fato instável.

Definição. Diremos que o lagrangeano $\mathcal{L}(q, \dot{q})=T(q, \dot{q})-\pi(q)$ satisfaz as hipóteses $\mathcal{H}_{1}$ se valerem:

1. $\mathcal{L}=T-\pi$ é de classe $\mathcal{C}^{2}$ e está definido para q pertencente a alguma vizinhança aberta de 0 em $\mathbb{R}^{n}$ e $\dot{q} \in \mathbb{R}^{n}$;

2. T é uma forma quadrática definida positiva em $\dot{q}$ para todo $q$ e $T(0, \dot{q})=\frac{1}{2} \sum_{i=1}^{n} \dot{q}_{i}^{2}$; 
3. existem naturais $N$ e $k \geq 2$ tal que $\pi\left(q_{1}, \ldots, q_{n}\right)=U\left(q_{1}, \ldots, q_{N}\right)+\pi_{2}\left(q_{N+1}, \ldots, q_{n}\right)$, sendo que:

(a) $U\left(q_{1}, \ldots, q_{N}\right) \geq 0$ para todo $\left(q_{1}, \ldots, q_{N}\right)$;

(b) $\pi(0)=\left\|\frac{\partial \pi}{\partial q}(0)\right\|=0$;

(c) $j^{k} \pi_{2}$ mostra que $\pi_{2}$ não tem mínimo na origem;

(d) existe $j^{k-1} \nabla \pi_{2}$ na origem.

Devemos notar que, com estas hipóteses, temos naturalmente que $\pi_{2}=j^{k} \pi_{2}+R$, $\operatorname{com} R\left(q_{N+1}, \ldots, q_{n}\right)=o\left(\left\|\left(q_{N+1}, \ldots, q_{n}\right)\right\|^{k}\right)$. Ainda, como existe $j^{k-1} \nabla \pi_{2}$, demonstrase no apêndice de Garcia e Tal (2003) que $\frac{\partial R}{\partial q_{j}}=o\left(\left\|\left(q_{N+1}, \ldots, q_{n}\right)\right\|^{k-1}\right)$ para $j=$ $N+1, \ldots, n$. 


\section{Capítulo 2}

\section{Lemas e estimativas iniciais}

Mantemos aqui a notação dada no capítulo anterior e supomos que $\mathcal{L}$ satisfaz $\mathcal{H}_{0}$. Em particular, $\delta, \sigma$ e $C$ são os dados pela propriedade- $(\alpha, \beta)$ de $P$ e $E(q, \dot{q})=T(q, \dot{q})+$ $\pi(q)$ é a energia total do sistema.

Nessas condições, podemos escrever as equações de Lagrange em coordenadas e, após um breve desenvolvimento, chegar a

$$
\frac{d}{d t}\left(\sum_{s=1}^{n} g_{r s} \dot{q}_{s}\right)=-\frac{\partial \pi}{\partial q_{r}}+\frac{1}{2} \sum_{l, s=1}^{n} \frac{\partial g_{l s}}{\partial q_{r}} \dot{q}_{l} \dot{q}_{s}
$$

com $r=1, \ldots, n$, que apesar de não estarem na sua forma normal, será a forma mais conveniente na qual as utilizaremos adiante.

Com estas hipóteses, podemos fazer uma pequena modificação na função auxiliar usada em Garcia e Tal (2003), que preservará as boas propriedades da mesma. Ou seja, como veremos no que segue, obteremos as mesmas estimativas obtidas em Garcia e Tal (2003) sobre a função auxiliar que nos permitirá, então, seguir o mesmo roteiro para provar o teorema de instabilidade. 
Seja $p_{n}(q, \dot{q})=\sum_{s=1}^{n} g_{n s} \dot{q}_{s}$ e considere

$$
V(q, \dot{q})=\alpha q_{n}^{\beta}-\frac{1}{2 g_{n n}} p_{n}^{2}-R\left(q_{N+1}, \ldots, q_{n}\right)
$$

e $\widetilde{V}(q, \dot{q})=\frac{V(q, \dot{q})}{q_{n}^{g}}$. Definimos então, para $\sigma_{1} \in(0, \sigma)$ e $\lambda>0$

$$
C_{\sigma_{1}}=\left\{(q, \dot{q}): \begin{array}{cc}
E(q, \dot{q})=0, & \left(q_{N+1}, \ldots, q_{n}\right) \in C, \\
\widetilde{V}(q, \dot{q})<\delta & \text { e } q_{n} \in\left(0, \sigma_{1}\right)
\end{array}\right\}
$$

e

$$
C_{\sigma_{1}, \lambda}=C_{\sigma_{1}} \cap\left\{(q, \dot{q}):\left\|\left(q_{1}, \ldots, q_{n-1}\right)\right\|_{\infty}<\lambda q_{n}\right\}
$$

Notamos que $C_{\sigma_{1}}$ não é vazio pois todo ponto tal que $\frac{g_{n n}}{2} \dot{q}_{n}^{2}=\alpha q_{n}^{\beta}-R$, com as demais coordenadas sendo nulas e $q_{n}$ suficientemente pequeno está no conjunto.

Como buscamos órbitas assintóticas à origem, e pela definição de $C_{\sigma_{1}}$, faz sentido trabalharmos na topologia relativa do nível de energia $E=0$. Desta forma, todas as operações de conjuntos que realizarmos a partir daqui serão nesta topologia relativa.

Vale notar que, como $E=T+U-\alpha q_{n}^{\beta}+P+R$, em $C_{\sigma_{1}}$ temos que $V=T-$ $\frac{1}{2 g_{n n}} p_{n}^{2}+U+P$. Como $T(0, \dot{q})=\frac{1}{2} \sum_{i=1}^{n} \dot{q}_{i}^{2}$ e $T(q, \dot{q})-\frac{1}{2 g_{n n}} p_{n}^{2}(q, \dot{q})$ não possui termos em $\dot{q}_{n}$, dado $\varepsilon>0$, para $\|q\|$ suficientemente pequena, temos que $\frac{(1-\varepsilon)}{2} \sum_{i=1}^{n-1} \dot{q}_{i}^{2} \leq$ $T(q, \dot{q})-\frac{1}{2 g_{n n}} p_{n}^{2}(q, \dot{q}) \leq \frac{(1+\varepsilon)}{2} \sum_{i=1}^{n-1} \dot{q}_{i}^{2}$.

Assim, lembrando que $U$ e $P$ são positivas, temos que

$$
0 \leq \frac{(1-\varepsilon)}{2} \sum_{i=1}^{n-1} \dot{q}_{i}^{2} \leq \frac{(1-\varepsilon)}{2} \sum_{i=1}^{n-1} \dot{q}_{i}^{2}+U+P \leq V<\delta q_{n}^{\beta}
$$


Daqui, segue a importante estimativa que vale em $C_{\sigma_{1}, \lambda}$ :

$$
\dot{q}_{j}^{2}=O\left(q_{n}^{\beta}\right)
$$

para $j=1, \ldots, n-1$.

Provamos agora uma limitação importante para $\dot{q}_{n}$, que será utilizada logo em seguida para determinar o sinal de $\dot{\widetilde{V}}$.

Lema 1. Sejam $\lambda>0$ e $\varepsilon \in(0,1)$. Então existe $\sigma_{0}>0$ tal que se $(q, p) \in \bar{C}_{\sigma_{0}, \lambda}$, então

$$
\frac{\alpha}{1+\varepsilon} q_{n}^{\beta} \leq \dot{q}_{n}^{2} \leq \frac{4 \alpha}{1-\varepsilon} q_{n}^{\beta}
$$

Demonstração. Existe $\sigma_{1}>0$ tal que, pelas hipóteses sobre $g_{l s}$ e pela equação (2.3), em $C_{\sigma_{1}, \lambda}$ temos

$$
\frac{1-\varepsilon}{2} \dot{q}_{n}^{2}+o\left(q_{n}^{\beta}\right) \leq \frac{1}{2 g_{n n}}\left(\sum_{s=1}^{n} g_{n s} \dot{q}_{s}\right)^{2} \leq \frac{1+\varepsilon}{2} \dot{q}_{n}^{2}+o\left(q_{n}^{\beta}\right)
$$

Considere $\sigma_{2} \in\left(0, \sigma_{1}\right]$ tal que, como observamos após a definição de $V$, temos $V \geq 0$ em $C_{\sigma_{2}, \lambda}$. Da hipótese sobre a ordem de $R$, temos que que $R=o\left(q_{n}^{\beta}\right)$ em $C_{\sigma_{2}, \lambda}$, e usando a definição de $V$ temos

$$
\alpha q_{n}^{\beta}-\frac{1}{2 g_{n n}} p_{n}^{2}+o\left(q_{n}^{\beta}\right) \geq 0,
$$

de onde $\frac{1}{2 g_{n n}} p_{n}^{2} \leq \alpha q_{n}^{\beta}+o\left(q_{n}^{\beta}\right)$. Usando (2.4), obtemos $\frac{1-\varepsilon}{2} \dot{q}_{n}^{2} \leq \alpha q_{n}^{\beta}+o\left(q_{n}^{\beta}\right)$. Daqui segue que $\dot{q}_{n}^{2} \leq \frac{2 \alpha}{1-\varepsilon} q_{n}^{\beta}+o\left(q_{n}^{\beta}\right)$ e, para um $\sigma_{3} \in\left(0, \sigma_{2}\right]$ obtemos $\dot{q}_{n}^{2} \leq \frac{4 \alpha}{1-\varepsilon} q_{n}^{\beta}$ em $C_{\sigma_{3}, \lambda}$.

Por outro lado, temos $V<\delta q_{n}^{\beta}$ em $C_{\sigma_{3, \lambda}}$ e, da definição de $V$ e ordem de $R$ temos

$$
\alpha q_{n}^{\beta}-\frac{1}{2 g_{n n}} p_{n}^{2}+o\left(q_{n}^{\beta}\right)<\delta q_{n}^{\beta},
$$


de onde $\frac{1}{2 g_{n n}} p_{n}^{2}>(\alpha-\delta) q_{n}^{\beta}+o\left(q_{n}^{\beta}\right)$. Novamente por (2.4), e lembrando que da propriedade- $(\alpha, \beta)$, temos $\delta<\frac{1}{4} \alpha$, obtemos $\frac{1+\varepsilon}{2} \dot{q}_{n}^{2} \geq(\alpha-\delta) q_{n}^{\beta}+o\left(q_{n}^{\beta}\right) \geq \frac{3}{4} \alpha q_{n}^{\beta}+o\left(q_{n}^{\beta}\right)$, de onde segue que $(1+\varepsilon) \dot{q}_{n}^{2} \geq \frac{3}{2} \alpha q_{n}^{\beta}+o\left(q_{n}^{\beta}\right)$. Finalmente, podemos tomar um $\sigma_{0}\left(0, \sigma_{3}\right]$ tal que, em $C_{\sigma_{0}, \lambda}$ temos $\dot{q}_{n}^{2} \geq \frac{\alpha}{(1+\varepsilon)} q_{n}^{\beta}$.

Faremos agora a importante determinação do sinal de $\dot{\widetilde{V}}$, antes de provar o teorema de instabilidade. Lembramos que o bordo do conjunto descrito no lema a seguir está sendo tomado na topologia relativa do nível $E=0$.

Lema 2. Para todo $\lambda>0$, existe $\sigma_{0} \in(0, \sigma)$ tal que em

$$
\left(\partial C_{\sigma_{0}} \backslash\{(0,0)\}\right) \cap\left\{(q, \dot{q}):\left\|\left(q_{1}, \ldots, q_{n-1}\right)\right\|_{\infty} \leq \lambda q_{n}\right\}
$$

a função $\dot{\widetilde{V}}$ não se anula. Mais precisamente, $\dot{\widetilde{V}}$ tem o sinal oposto ao de $\dot{q}_{n}$ em cada componente conexa desse conjunto .

Demonstração. Como $\widetilde{V}=\frac{V}{q_{n}^{\beta}}$, temos que $\dot{\widetilde{V}}=\frac{\dot{V}}{q_{n}^{\beta}}-\frac{\beta V}{q_{n}^{\beta+1}} \dot{q}_{n}$, e portanto

$$
q_{n}^{\beta+1} \dot{\widetilde{V}}=q_{n} \dot{V}-\beta V \dot{q}_{n}
$$

Como $q_{n}>0$ em $C_{\sigma, \lambda}$, basta mostrar que $q_{n}^{\beta+1} \dot{\tilde{V}}$ não se anula.

Calculemos $\dot{V}$, usando a definição de $V$

$$
\dot{V}=\alpha \beta q_{n}^{\beta-1} \dot{q}_{n}-\frac{1}{g_{n n}} p_{n} \dot{p}_{n}+\frac{1}{2 g_{n n}^{2}} \dot{g}_{n n} p_{n}^{2}-\sum_{i=N+1}^{n} \frac{\partial R}{\partial q_{i}} \dot{q}_{i} .
$$


Daqui, usando a definição de $p_{n}$ temos

$$
\begin{aligned}
\dot{V}= & \alpha \beta q_{n}^{\beta-1} \dot{q}_{n}-\frac{1}{g_{n n}}\left(\sum_{s=1}^{n} g_{n s} \dot{q}_{s}\right) \frac{d}{d t}\left(\sum_{s=1}^{n} g_{n s} \dot{q}_{s}\right) \\
& +\frac{1}{2 g_{n n}^{2}} \dot{g}_{n n}\left(\sum_{s=1}^{n} g_{n s} \dot{q}_{s}\right)^{2}-\sum_{i=N+1}^{n} \frac{\partial R}{\partial q_{i}} \dot{q}_{i}
\end{aligned}
$$

e usando as equações (2.1), obtemos

$$
\begin{aligned}
\dot{V}= & \alpha \beta q_{n}^{\beta-1} \dot{q}_{n}-\frac{\left(\sum_{s=1}^{n} g_{n s} \dot{q}_{s}\right)}{g_{n n}}\left(-\frac{\partial \pi}{\partial q_{n}}+\frac{1}{2} \sum_{l, s=1}^{n} \frac{\partial g_{l s}}{\partial q_{n}} \dot{q}_{l} \dot{q}_{s}\right) \\
& +\frac{1}{2 g_{n n}^{2}} \dot{g}_{n n}\left(\sum_{s=1}^{n} g_{n s} \dot{q}_{s}\right)^{2}-\sum_{i=N+1}^{n} \frac{\partial R}{\partial q_{i}} \dot{q}_{i} .
\end{aligned}
$$

Usando então as hipóteses sobre a forma de $\pi$, temos

$$
\begin{aligned}
\dot{V}= & \alpha \beta q_{n}^{\beta-1} \dot{q}_{n}-\frac{\left(\sum_{s=1}^{n} g_{n s} \dot{q}_{s}\right)}{g_{n n}}\left(\alpha \beta q_{n}^{\beta-1}-\frac{\partial P}{\partial q_{n}}-\frac{\partial R}{\partial q_{n}}\right) \\
& -\frac{\left(\sum_{s=1}^{n} g_{n s} \dot{q}_{s}\right)}{2 g_{n n}}\left(\sum_{l, s=1}^{n} \frac{\partial g_{l s}}{\partial q_{n}} \dot{q}_{l} \dot{q}_{s}\right)+\frac{1}{2 g_{n n}^{2}} \dot{g}_{n n}\left(\sum_{s=1}^{n} g_{n s} \dot{q}_{s}\right)^{2} \\
& -\sum_{i=N+1}^{n} \frac{\partial R}{\partial q_{i}} \dot{q}_{i}
\end{aligned}
$$




$$
\begin{aligned}
\dot{V}= & -\frac{\left(\sum_{s=1}^{n-1} g_{n s} \dot{q}_{s}\right)}{g_{n n}}\left(\alpha \beta q_{n}^{\beta-1}\right)+\frac{\left(\sum_{s=1}^{n} g_{n s} \dot{q}_{s}\right)}{g_{n n}} \frac{\partial P}{\partial q_{n}} \\
& +\frac{\left(\sum_{s=1}^{n} g_{n s} \dot{q}_{s}\right)}{g_{n n}} \frac{\partial R}{\partial q_{n}}-\frac{\left(\sum_{s=1}^{n} g_{n s} \dot{q}_{s}\right)}{2 g_{n n}}\left(\sum_{l, s=1}^{n} \frac{\partial g_{l s}}{\partial q_{n}} \dot{q}_{l} \dot{q}_{s}\right) \\
& +\frac{1}{2 g_{n n}^{2}} \dot{g}_{n n}\left(\sum_{s=1}^{n} g_{n s} \dot{q}_{s}\right)^{2}-\sum_{i=N+1}^{n} \frac{\partial R}{\partial q_{i}} \dot{q}_{i}, \\
\dot{V}= & -\frac{\left(\sum_{s=1}^{n-1} g_{n s} \dot{q}_{s}\right)}{g_{n n}}\left(\alpha \beta q_{n}^{\beta-1}-\frac{\partial P}{\partial q_{n}}\right)+\dot{q}_{n} \frac{\partial P}{\partial q_{n}} \\
& +\frac{\left(\sum_{s=1}^{n} g_{n s} \dot{q}_{s}\right)}{g_{n n}} \frac{\partial R}{\partial q_{n}}-\frac{\left(\sum_{s=1}^{n} g_{n s} \dot{q}_{s}\right)}{2 g_{n n}}\left(\sum_{l, s=1}^{n} \frac{\partial g_{l s}}{\partial q_{n}} \dot{q}_{l} \dot{q}_{s}\right) \\
& +\frac{1}{2 g_{n n}^{2}} \dot{g}_{n n}\left(\sum_{s=1}^{n} g_{n s} \dot{q}_{s}\right)^{2}-\sum_{i=N+1}^{n} \frac{\partial R}{\partial q_{i}} \dot{q}_{i} .
\end{aligned}
$$

Assim, podemos escrever

$$
\begin{aligned}
q_{n} \dot{V}= & -\frac{\left(\sum_{s=1}^{n-1} g_{n s} \dot{q}_{s}\right)}{g_{n n}}\left(\alpha \beta q_{n}^{\beta}-q_{n} \frac{\partial P}{\partial q_{n}}\right)+\dot{q}_{n} q_{n} \frac{\partial P}{\partial q_{n}} \\
& +q_{n} \frac{\left(\sum_{s=1}^{n} g_{n s} \dot{q}_{s}\right)}{g_{n n}} \frac{\partial R}{\partial q_{n}}-q_{n} \frac{\left(\sum_{s=1}^{n} g_{n s} \dot{q}_{s}\right)}{2 g_{n n}}\left(\sum_{l, s=1}^{n} \frac{\partial g_{l s}}{\partial q_{n}} \dot{q}_{l} \dot{q}_{s}\right) \\
& +\frac{q_{n}}{2 g_{n n}^{2}} \dot{g}_{n n}\left(\sum_{s=1}^{n} g_{n s} \dot{q}_{s}\right)^{2}-q_{n}\left(\sum_{i=N+1}^{n} \frac{\partial R}{\partial q_{i}} \dot{q}_{i}\right) .
\end{aligned}
$$

Com exceção do termo $\dot{q}_{n} q_{n} \frac{\partial P}{\partial q_{n}}$, para $\sigma_{0}>0$ suficientemente pequeno, os demais termos de $q_{n} \dot{V}$ são $o\left(q_{n}^{\frac{3 \beta}{2}}\right)$. De fato, notando que por hipótese temos que $g_{i j} \rightarrow 0$ se $i \neq j$ e $g_{i i} \rightarrow 1$, analisemos cada um dos demais termos: 
- $\frac{\left(\sum_{s=1}^{n-1} g_{n s} \dot{q}_{s}\right)}{g_{n n}} \alpha \beta q_{n}^{\beta}$ : lembrando que $\dot{q}_{j}=O\left(q_{n}^{\frac{\beta}{2}}\right)$ por $(2.3)$, temos que cada parcela é $O\left(q_{n}^{\frac{3 \beta}{2}}\right)$

- $\frac{\left(\sum_{s=1}^{n-1} g_{n s} \dot{q}_{s}\right)}{g_{n n}} q_{n} \frac{\partial P}{\partial q_{n}}$ : por $(2.3)$ e pela propriedade- $(\alpha, \beta)$ de $P$, concluímos que cada parcela é $o\left(q_{n}^{\frac{3 \beta}{2}}\right)$;

- $q_{n} \frac{\left(\sum_{s=1}^{n} g_{n s} \dot{q}_{s}\right)}{g_{n n}} \frac{\partial R}{\partial q_{n}}$ e $q_{n}\left(\sum_{i=N+1}^{n} \frac{\partial R}{\partial q_{i}} \dot{q}_{i}\right)$ : das hipóteses para $R$, temos que $\frac{\partial R}{\partial q_{n}}=$ $o\left(q_{n}^{\beta-1}\right)$ e através de $(2.3)$ e do lema 1 concluímos que neste termo também cada parcela é $o\left(q_{n}^{\frac{3 \beta}{2}}\right)$;

- $q_{n} \frac{\left(\sum_{s=1}^{n} g_{n s} \dot{q}_{s}\right)}{2 g_{n n}}\left(\sum_{l, s=1}^{n} \frac{\partial g_{l s}}{\partial q_{n}} \dot{q}_{l} \dot{q}_{s}\right):$ do fato que por hipótese $\frac{\partial g_{l s}}{\partial q_{n}}$ tem ordem $\mu_{2}>-1$, de onde $q_{n} \frac{\partial g_{l_{s}}}{\partial q_{n}}$ tem ordem estritamente positiva, segue de (2.3) e do lema 1 que cada parcela é $o\left(q_{n}^{\frac{3 \beta}{2}}\right)$;

- $\frac{q_{n}}{2 g_{n n}^{2}} \dot{g}_{n n}\left(\sum_{s=1}^{n} g_{n s} \dot{q}_{s}\right)^{2}$ : notando que $\dot{g}_{n n}=\sum_{i=1}^{n} \frac{\partial g_{n n}}{\partial q_{i}} \dot{q}_{i}$ e que por hipótese $\frac{\partial g_{l s}}{\partial q_{n}}$ tem ordem $\mu_{2}>-1$, de onde $q_{n} \frac{\partial g_{l s}}{\partial q_{n}}$ tem ordem estritamente positiva, segue de (2.3) e do lema 1 que cada parcela é $o\left(q_{n}^{\frac{3 \beta}{2}}\right)$.

Com isso, obtemos

$$
q_{n} \dot{V}=\dot{q}_{n} q_{n} \frac{\partial P}{\partial q_{n}}+o\left(q_{n}^{\frac{3 \beta}{2}}\right)
$$

Notamos então que em $\partial C_{\sigma_{0}, \lambda}$ temos que $V=\delta q_{n}^{\beta}$, e $\operatorname{assim} \beta V \dot{q}_{n}=\beta \delta q_{n}^{\beta} \dot{q}_{n}$. Com isso, substituindo os resultados na equação (2.5), temos que

$$
\begin{aligned}
q_{n}^{\beta+1} \dot{\widetilde{V}} & =\dot{q}_{n} q_{n} \frac{\partial P}{\partial q_{n}}-\beta \delta q_{n}^{\beta} \dot{q}_{n}+o\left(q_{n}^{\frac{3 \beta}{2}}\right) \\
& =-\left(\beta \delta q_{n}^{\beta}-q_{n} \frac{\partial P}{\partial q_{n}}\right) \dot{q}_{n}+o\left(q_{n}^{\frac{3 \beta}{2}}\right)
\end{aligned}
$$


Como em $\partial C_{\sigma_{0}, \lambda}$ temos que $P \leq \delta q_{n}^{\beta}$, pela propriedade- $(\alpha, \beta)$ de $P$ temos que $q_{n} \frac{\partial P}{\partial q_{n}} \leq \delta(k-1) q_{n}^{\beta}$. Assim

$$
\beta \delta q_{n}^{\beta}-q_{n} \frac{\partial P}{\partial q_{n}} \geq(\beta-(k-1)) \delta q_{n}^{\beta} .
$$

Lembrando que $(\beta-(k-1))>0$, que para $\sigma_{0}$ dado pelo lema 1 suficientemente pequeno, temos $\dot{q}_{n} \neq 0$ e pelas desigualdades do mesmo lema 1, temos

$$
\begin{aligned}
\left|q_{n}^{\beta+1} \dot{\tilde{V}}\right| & \geq(\beta-(k-1)) \delta q_{n}^{\beta}\left|\dot{q}_{n}\right|-\left|o\left(q_{n}^{\frac{3 \beta}{2}}\right)\right| \\
& \geq(\beta-(k-1)) \delta \sqrt{\alpha} q_{n}^{\frac{3 \beta}{2}}-\left|o\left(q_{n}^{\frac{3 \beta}{2}}\right)\right|
\end{aligned}
$$

Finalmente, diminuindo $\sigma_{0}$ se necessário, o resultado segue. Em particular, a equação (2.6) mostra que o sinal de $\dot{\widetilde{V}}$ em

$$
\left(\partial C_{\sigma_{0}} \backslash\{(0,0)\}\right) \cap\left\{(q, \dot{q}):\left\|\left(q_{1}, \ldots, q_{n-1}\right)\right\|_{\infty} \leq \lambda q_{n}\right\}
$$

é dado pelo sinal oposto do sinal $\dot{q}_{n}$, como queríamos. 


\section{Capítulo 3}

\section{Teorema de instabilidade}

Provamos agora o resultado central desse texto, que é o teorema de instabilidade e existência de uma órbita assintótica à origem com $t \rightarrow-\infty$, o que garante a instabilidade do ponto de equilíbrio. A demonstração, agora que obtivemos as estimativas dos lemas 1 e 2, é uma extensão simples e direta do teorema análogo em Garcia e Tal (2003).

Teorema 1. Considere o sistema lagrangeano dado pelas equações (2.1). Admita ainda que $\mathcal{L}$ satisfaz $\mathcal{H}_{0}$. Então, existe $\rho>0$ e uma trajetória $\phi(t)$ tal que $(\phi(t), \dot{\phi}(t)) \rightarrow(0,0)$ com $t \rightarrow-\infty$, e que para $|t|$ suficientemente grande, $(\phi(t), \dot{\phi}(t)) \in C_{\rho, \sqrt{2 \alpha}}$.

Demonstração. Dado $\gamma>0$, vamos denotar por $\Omega_{\gamma}$ o conjunto

$$
\Omega_{\gamma}=C_{\gamma, \sqrt{2 \alpha}} \cap\left\{(q, \dot{q}): \dot{q}_{n}>0\right\}
$$

Pelo lema 1 , existe $\rho_{1}>0$ tal que em $\Omega_{\rho_{1}}, \dot{q}_{n}^{2}>\frac{1}{2} \alpha q_{n}^{\beta}$. Como $\dot{q}_{n}>0$ temos que $\dot{q}_{n}>\sqrt{\frac{1}{2} \alpha} q_{n}^{\frac{\beta}{2}}$. 
Ainda, da equação (2.2), temos que existe $\rho_{2} \in\left(0, \rho_{1}\right]$ tal que em $\Omega_{\rho_{2}}$

$$
\dot{q}_{j}^{2}<4 \delta q_{n}^{\beta}<2 \alpha \dot{q}_{n}^{2}
$$

para $j=1, \ldots, n-1$.

Assim, para qualquer solução $\varphi(t)=\left(q_{1}(t), \ldots, q_{n}(t)\right)$ de $(2.1)$, se existe um $t_{1}$ tal que $\left(\varphi\left(t_{1}\right), \dot{\varphi}\left(t_{1}\right)\right) \in \bar{\Omega}_{\rho_{2}}$ e $\left|q_{j}\left(t_{1}\right)\right|=q_{n}\left(t_{1}\right)$, então existe $\epsilon_{1}>0$ tal que para todo $t \in\left(t_{1}, t_{1}+\epsilon_{1}\right)$ temos que $\left|q_{j}(t)\right|<\sqrt{2 \alpha} q_{n}(t)$ e para todo $t \in\left(t_{1}-\epsilon, t_{1}\right)$, temos $(\varphi(t), \dot{\varphi}(t)) \notin \bar{\Omega}_{\rho_{2}}$.

Por outro lado, o lema 2 implica que existe $\rho \in\left(0, \rho_{2}\right]$ tal que se $\left(\varphi\left(t_{2}\right), \dot{\varphi}\left(t_{2}\right)\right) \in \bar{\Omega}_{\rho}$ e $V\left(\varphi\left(t_{2}\right), \dot{\varphi}\left(t_{2}\right)\right)=\delta q_{n}\left(t_{2}\right)^{\beta}$ para algum instante $t_{2}$, então existe $\epsilon_{2}>0$ tal que para todo $t \in\left(t_{2}, t_{2}+\epsilon_{2}\right)$ temos $V(\varphi(t), \dot{\varphi}(t))<\delta q_{n}(t)^{\beta}$ e para todo $t \in\left(t_{2}-\epsilon_{2}, t_{2}\right)$ temos $V(\varphi(t), \dot{\varphi}(t))>\delta q_{n}(t)^{\beta}$.

Portanto, cada solução $\varphi$, com $0<q_{n}(t)<\rho$, que em algum instante $\tilde{t}$ está no bordo de $\Omega_{\rho}$ tem que ter estado fora de $\Omega_{\rho}$ algum intervalo de tempo antes de $\tilde{t}$. E, além disso, essa solução vai estar no interior relativo de $\Omega_{\rho}$ por um intervalo de tempo posterior a $\tilde{t}$.

Tomamos então uma seqüência $z_{j}=\left(q_{1, j}, \ldots, q_{n, j}, \dot{q}_{1, j}, \ldots, \dot{q}_{n, j}\right) \in \partial \Omega_{\rho}$, com $0<$ $q_{n, j}<\rho$ e tal que $\lim _{j \rightarrow \infty} z_{j}=(0,0)$ e considere as soluções $\phi_{j}$ de $(2.1)$ tais que

$$
\left(\phi_{j}(0), \dot{\phi}_{j}(0)\right)=z_{j}
$$

Em algum instante positivo, essas soluções vão estar em $\Omega_{\rho}$ e, também, não podem sair de $\Omega_{\rho}$ em um ponto com $q_{n}<\rho$. Ainda, como em $\Omega_{\rho}$ temos $\dot{q}_{n}>\sqrt{\frac{1}{2} \alpha} q_{n}^{\frac{\beta}{2}}$, existem seqüências $t_{j}>0$ e $w_{j}=\left(\tilde{q}_{1, j}, \ldots, \rho, \dot{\tilde{q}}_{1, j}, \ldots, \dot{\tilde{q}}_{n, j}\right) \in \partial \Omega_{\rho}$, tais que $\left(\phi_{j}\left(t_{j}\right), \dot{\phi}_{j}\left(t_{j}\right)\right)=w_{j}$ 
e $\left(\phi_{j}(t), \dot{\phi}_{j}(t)\right) \in \Omega_{\rho}$ para todo $t \in\left(0, t_{j}\right)$.

É claro que existe uma subseqüência $w_{j_{i}}$ convergente a um ponto $\bar{w}$. Sem perda de generalidade, vamos supor $w_{j_{i}}=w_{j}$. É claro que $\bar{w} \neq 0$, dado que a $n$-ésima coordenada de $w_{j}$ é sempre $\rho$.

Afirmamos que a solução $\phi(t)$ de $(2.1)$ de condições iniciais $\bar{w}$ é assintótica a $(0,0)$ com $t \rightarrow-\infty$.

Suponha por contradição que isso não ocorre. Então, existiria um instante $\bar{t}<0$ tal que $(\phi(\bar{t}), \dot{\phi}(\bar{t})) \notin \bar{\Omega}_{\rho}$.

Seja

$$
d=\left\{\operatorname{dist}\left((\phi(\bar{t}), \dot{\phi}(\bar{t})), \partial \Omega_{\rho}\right), \min _{t \in[\bar{t}, 0]}\|(\phi(t), \dot{\phi}(t))\|\right\},
$$

com $\operatorname{dist}\left(\left(\phi(\bar{t}), \dot{\phi}(\bar{t}), \partial \Omega_{\rho}\right)\right.$ sendo a distância de $(\phi(\bar{t}), \dot{\phi}(\bar{t}))$ a $\partial \Omega_{\rho}$.

Como $w_{j}$ converge para $\bar{w}$, a dependência contínua garante que existe $j_{0}$ tal que, para $j>j_{0}$ temos

$$
\min _{t \in[\bar{t}, 0]}\left\|(\phi(t), \dot{\phi}(t))-\left(\phi_{j}\left(t_{j}+t\right), \dot{\phi}_{j}\left(t_{j}+t\right)\right)\right\|<\frac{d}{2},
$$

o que implica que $t_{j}+\bar{t}<0$ para $j>j_{0}$, pois

$$
\left\|(\phi(\bar{t}), \dot{\phi}(\bar{t}))-\left(\phi_{j}\left(t_{j}+\bar{t}\right), \dot{\phi}_{j}\left(t_{j}+\bar{t}\right)\right)\right\|<\frac{d}{2} \Longrightarrow\left(\left(\phi_{j}\left(t_{j}+\bar{t}\right), \dot{\phi}_{j}\left(t_{j}+\bar{t}\right)\right) \notin \bar{\Omega}_{\rho} .\right.
$$

Mas então temos que $z_{j}=\left(\phi_{j}(0), \dot{\phi}_{j}(0)\right)=\left(\phi_{j}\left(t_{j}+\left(-t_{j}\right)\right), \dot{\phi}_{j}\left(t_{j}+\left(-t_{j}\right)\right)\right)$ não tende a $(0,0)$ quando $j \rightarrow \infty$, o que é absurdo pela escolha de $z_{j}$ e completa a demonstração. 


\section{Capítulo 4}

\section{O splitting com o plano}

Neste capítulo analisamos um caso particular de sistemas lagrangeanos em que temos uma mudança de coordenadas que nos leva a um sistema que satisfaz as hipóteses $\mathcal{H}_{0}$. Assim, aplicando o teorema 1, obtemos um teorema de instabilidade para este caso.

O problema inicial que motiva este capítulo é tentar obter uma generalização do resultado principal de Garcia e Tal (2003) em um splitting de $\mathbb{R}^{n}$, analogamente embora com técnicas muito distintas - ao que é feito em Maffei, Moauro e Negrini (1991) para estender os resultados de Moauro e Negrini (1989).

Desta forma, supomos aqui que estudamos o sistema $(2.1)$, com lagrangeano $\mathcal{L}=$ $T-\pi$ satisfazendo $\mathcal{H}_{1}$ e admitimos que $n-N=2$, ou seja, $\pi_{2}=\pi_{2}\left(q_{n-1}, q_{n}\right)$ é uma função de duas variáveis, cujo jato $k$ mostra que ela não tem mínimo na origem.

Então, conforme a seção 3 de Garcia e Tal (2003), existe uma mudança de coordenadas nas quais $\pi_{2}$ pode ser escrita de tal forma que satisfaz $\mathcal{H}_{0}$. Em particular, o lema 1 de Garcia e Tal (2003) demonstra a propriedade- $(\alpha, \beta)$ de $P$.

Para completar a demonstração, basta verificarmos que a energia cinética, nas novas coordenadas, satisfaz as hipóteses sobre sua ordem. Para isso, relembremos brevemente 
como é construída a mudança de coordenadas feita em Garcia e Tal (2003).

Suponha, inicialmente, que $j^{k} \pi_{2}$ não é homogêneo ou é homogêneo e existe $\left(q_{n-1}, q_{n}\right)$ tal que $j^{k} \pi_{2}\left(q_{n-1}, q_{n}\right)>0$, isto é, trata-se de uma sela homogênea. Nessas condições, demonstra-se no apêndice de Garcia e Tal (2003) que existem reais $\sigma, \lambda_{0}, \alpha>0,(k-$ 1) $<\beta \leq k$ e uma curva algébrica $\gamma:[0, \sigma) \rightarrow \mathbb{R}$, com $\gamma(0)=0$, tal que, após uma eventual rotação, vale

$$
\min _{-\lambda_{0} q_{n}<q_{n-1}<\lambda_{0} q_{n}} j^{k} \pi_{2}\left(q_{n-1}, q_{n}\right)=j^{k} \pi_{2}\left(\gamma\left(q_{n}\right), q_{n}\right)=-\alpha q_{n}^{\beta}+o\left(q_{n}^{\beta}\right),
$$

para todo $q_{n} \in[0, \sigma)$.

Além disso, temos que $\gamma\left(q_{n}\right)=\sum_{i=1}^{\infty} b_{i} q_{n}^{\beta_{i}}$, com $b_{i} \in \mathbb{R}$ e $\left(\beta_{i}\right)$ uma seqüência estritamente crescente de racionais, $\operatorname{com} \beta_{1}>1$. Daqui segue que, com $\sigma$ eventualmente menor, existem constantes positivas $c_{1}, c_{2}$ e $c_{3}$ tais que, para $q_{n} \in[0, \sigma)$, temos

$$
\begin{aligned}
& \left|\gamma\left(q_{n}\right)\right|<c_{1} q_{n}^{\beta_{1}} \\
& \left|\gamma^{\prime}\left(q_{n}\right)\right|<c_{2} q_{n}^{\beta_{1}-1} \\
& \left|\gamma^{\prime \prime}\left(q_{n}\right)\right|<c_{3} q_{n}^{\beta_{1}-2} .
\end{aligned}
$$

A mudança de coordenadas que procuramos é feita em

$$
\mathcal{F}=\left\{\left(q_{n-1}, q_{n}\right): 0<q_{n}<\sigma\right\},
$$

onde será $\mathcal{C}^{\infty}$ e admitirá uma extensão a $\mathcal{F} \cup\{0\}$ que será um homeomorfismo, preservando as trajetórias assintóticas à origem tangentes ao semi-eixo $\left\{q_{n}>0\right\}$. Esta mudança, justamente, leva a curva $\gamma$ a este semi-eixo.

Assim, consideremos $\Phi\left(q_{n-1}, q_{n}\right)=\left(q_{n-1}-\gamma\left(q_{n}\right), q_{n}\right), \operatorname{com}\left(q_{n-1}, q_{n}\right) \in \mathcal{F}$ e $\Phi(0,0)=$ 
$(0,0)$. Nessas coordenadas, temos que $\pi_{2}$ satisfaz $\mathcal{H}_{0}$, o que é demonstrado em detalhes na seção 3 de Garcia e Tal (2003). Notamos que $P \geq 0$ para $\left|\tilde{q}_{n-1}\right| \leq \lambda_{0} q_{n}$, mas a demonstração do lema 1 em Garcia e Tal (2003) mostra que $P \geq 0$ em $C$, como desejamos.

Chamemos de $\Psi$ a mudança de variáveis em $\mathbb{R}^{n}$ tal que

$$
\Psi\left(q_{1}, \ldots, q_{n-1}, q_{n}\right)=\left(q_{1}, \ldots, q_{n-2}, \Phi\left(q_{n-1}, q_{n}\right)\right)
$$

Podemos, agora, calcular a energia cinética nas novas coordenadas e verificar que satisfaz as hipóteses desejadas sobre sua ordem.

Seja $q \in \mathbb{R}^{n}$ e denotemos as novas variáveis por $\tilde{q}=\left(q_{1}, \ldots, q_{n-1}-\gamma\left(q_{n}\right), q_{n}\right)$ e $\dot{\tilde{q}}=\left(\dot{q}_{1}, \ldots, \dot{q}_{n-1}-\gamma^{\prime}\left(q_{n}\right) \dot{q}_{n}, \dot{q}_{n}\right)$.

Sendo $T(q, \dot{q})=\frac{1}{2}\langle G(q) \dot{q}, \dot{q}\rangle$, temos então que nas novas coordenadas, a energia cinética $\tilde{T}$ é dada por

$$
\tilde{T}(\tilde{q}, \dot{\tilde{q}})=\frac{1}{2}\left\langle G\left(\Psi^{-1}(\tilde{q})\right) A(\tilde{q}) \dot{\tilde{q}}, A(\tilde{q}) \dot{\tilde{q}}\right\rangle
$$

onde $A(\tilde{q})$ é a matriz inversa da transformação induzida em $\dot{q}$ por $\Psi$, ou seja, $A^{-1}=\Psi^{\prime}$.

Desta forma, sendo $\tilde{G}(\tilde{q})=G\left(\Psi^{-1}(\tilde{q})\right)$, é claro que a matriz de $\tilde{T}$ é dada por $A^{\mathrm{T}} \tilde{G} A$. Basta agora calcularmos $A$. Para isso, notamos que

$$
\Psi^{\prime}=\left(\begin{array}{cc}
I_{N \times N} & 0 \\
0 & \Phi^{\prime}
\end{array}\right)
$$


onde, por sua vez, temos que

$$
\Phi^{\prime}=\left(\begin{array}{cc}
1 & -\gamma^{\prime} \\
0 & 1
\end{array}\right)
$$

cuja inversa é

$$
\left(\Phi^{\prime}\right)^{-1}=\left(\begin{array}{cc}
1 & \gamma^{\prime} \\
0 & 1
\end{array}\right)
$$

e, finalmente, é claro que

$$
A=\left(\Psi^{\prime}\right)^{-1}=\left(\begin{array}{cc}
I_{N \times N} & 0 \\
0 & \left(\Phi^{\prime}\right)^{-1}
\end{array}\right)
$$

de onde concluímos, através das relações (4.1), que $A^{\mathrm{T}} \tilde{G} A=I+h, \operatorname{com}\|h(\tilde{q})\|=$ $o\left(\|\tilde{q}\|^{\mu_{1}}\right)$ e $\left\|h^{\prime}(\tilde{q})\right\|=o\left(\|\tilde{q}\|^{\mu_{2}}\right), \operatorname{com} \mu_{1}=\min \left\{1, \beta_{1}-1\right\}>0$ e $\mu_{2}=\min \left\{0, \beta_{1}-2\right\}=$ $\mu_{1}-1>-1$, como desejávamos.

Com isso, através do teorema 1 provamos o seguinte:

Teorema 2. Considere o sistema lagrangeano dado pelas equações (2.1). Admita ainda que $\mathcal{L}$ satisfaz $\mathcal{H}_{1}$, que $n-N=2$ e que $j^{k} \pi_{2}$ na origem não é homogêneo ou é uma sela homogênea. Então existe uma trajetória $\phi(t)$ tal que $(\phi(t), \dot{\phi}(t)) \rightarrow(0,0)$ com $t \rightarrow-\infty$.

Em particular, este teorema em conjunto com o resultado de Maffei, Moauro e Negrini (1991) para o caso homogêneo, dá a extensão dos resultados de Garcia e Tal (2003), que citamos anteriormente como motivação, e apresentamos no corolário seguinte. Para isso, incluímos a hipótese do lagrangeano ser de uma classe de diferenciabilidade maior, que substitui a condição de acoplamento apresentada em Maffei, Moauro e Negrini (1991). O leitor interessado encontrará uma condição sutilmente mais fraca, que ga- 
ranta que o acoplamento do splitting é fraco, na mesma referência.

Corolário 1. Considere o sistema lagrangeano dado pelas equações (2.1). Admita $\mathcal{L}=T-\pi$ é tal que 0 é um ponto de equilíbrio e que existe um natural $k \geq 2$ tal que o jato $k$ de $\pi$ mostra que 0 não é um ponto de mínimo para $\pi$, que $\mathcal{L}$ é de classe

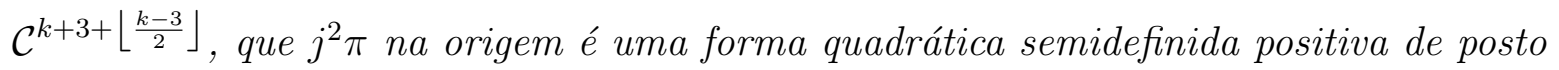
$n-2$ e que $T$ é uma forma quadrática positiva definida em $\dot{q}$ para todo q. Então existe uma trajetória $\phi(t)$ tal que $(\phi(t), \dot{\phi}(t)) \rightarrow(0,0)$ com $t \rightarrow-\infty$.

Demonstração. Como $\mathcal{L}$ é de classe pelo menos $\mathcal{C}^{k+3}$, segue que existe $j^{k-1} \nabla \pi$. E podemos, sem perda de generalidade, supor que $T(0, \dot{q})=\frac{\|\dot{q}\|^{2}}{2}$.

Então, basta aplicar o splitting lemma - conforme Barone Netto (1984) - e encontramos um sistema que satisfaz diretamente as hipóteses do teorema de Maffei, Moauro e Negrini (1991) ou do teorema 2, de onde o resultado segue facilmente. 


\section{Capítulo 5}

\section{Sistemas com forças giroscópicas}

Consideremos agora um sistema lagrangeano com a presença de forças não potenciais giroscópicas.

Mostramos que o teorema 1 pode ser estendido a este contexto, da mesma forma que em Moauro e Negrini (1989) generalizam-se os resultados de Furta (1986). Desta forma, graças ao capítulo anterior, temos uma generalização do teorema 2 e corolário 1 a este contexto também.

Lembramos que as equações de Lagrange para um sistema desses são

$$
\frac{d}{d t} \frac{\partial \mathcal{L}}{\partial \dot{q}}-\frac{\partial \mathcal{L}}{\partial q}=Q
$$

onde, por abuso de notação usual, $Q=Q(q, \dot{q})=Q(q) \dot{q}$ é linear em $\dot{q}$ e tal que $\langle Q(q) \dot{q}, \dot{q}\rangle=0$. Seus pontos de equilíbrio são da forma $\left(q_{0}, 0\right)$ tais que $\frac{\partial \pi}{\partial q}\left(q_{0}\right)=0$, como no caso conservativo.

Observamos que sendo $E(q, \dot{q})=T(q, \dot{q})+\pi(q)$ a energia do sistema, esta ainda é conservada na presença de forças giroscópias. Ou seja, temos que $\dot{E}=0$ em função de $Q$ satisfazer $\langle Q(q) \dot{q}, \dot{q}\rangle=0$. 
Assim, considerando-se que $\mathcal{L}$ satisfaça $\mathcal{H}_{0}$ e alguma hipótese adicional sobre $Q$, a construção feita para demonstrar o teorema 1 continua válida com pequenas mudanças. De fato, é simples notar que basta verificarmos a validade do lema 2 .

Podemos escrever as equações em coordenadas de modo conveniente, da mesma forma que as equações (2.1): considerando que a $j$-ésima componente de $Q(q) \dot{q}$ seja $\sum_{i=1}^{n} Q_{j i}(q) \dot{q}_{i}$, obtemos

$$
\frac{d}{d t}\left(\sum_{s=1}^{n} g_{r s} \dot{q}_{s}\right)=-\frac{\partial \pi}{\partial q_{r}}+\frac{1}{2} \sum_{l, s=1}^{n} \frac{\partial g_{l s}}{\partial q_{r}} \dot{q}_{l} \dot{q}_{s}+\sum_{i=1}^{n} Q_{r i}(q) \dot{q}_{i},
$$

$\operatorname{com} r=1, \ldots, n$.

Desta forma, mantendo as notações do caso conservativo, é fácil observar que no cálculo de $q_{n} \dot{V}$, graças às equações (5.1), apenas os seguintes termos adicionais aparecerão

$$
-q_{n} \frac{\left(\sum_{s=1}^{n} g_{n s} \dot{q}_{s}\right)}{g_{n n}} \sum_{i=1}^{n} Q_{n i}(q) \dot{q}_{i}
$$

que desejamos que sejam $o\left(q_{n}^{\frac{3 \beta}{2}}\right)$. Então, considerando que $\dot{q}_{j}=O\left(q_{n}^{\frac{\beta}{2}}\right)$, temos que basta que $Q_{n i}(q)=o\left(\|q\|^{\frac{\beta}{2}-1}\right)$ com $\|q\| \rightarrow 0$, para $i=1, \ldots, n$ e temos que vale o correspondente ao lema 2 e, consequentemente, ao teorema 1.

Assim, provamos o seguinte resultado.

Teorema 3. Considere o sistema lagrangeano com forças giroscópias $Q$ dado pelas equações (5.1). Admita ainda que $\mathcal{L}$ satisfaz $\mathcal{H}_{0}$ e que $Q$ é de classe $\mathcal{C}^{1}$ e $Q_{n i}(q)=$ $o\left(\|q\|^{\frac{\beta}{2}-1}\right)$ com $\|q\| \rightarrow 0$, para $i=1, \ldots, n$. Então, existe $\rho>0$ e uma trajetória $\phi(t)$ tal que $(\phi(t), \dot{\phi}(t)) \rightarrow(0,0)$ com $t \rightarrow-\infty$, e que para $|t|$ suficientemente grande, $(\phi(t), \dot{\phi}(t)) \in C_{\rho, \sqrt{2 \alpha}}$.

Para o caso em que $n-N=2$ e $\mathcal{L}$ satisfaz $\mathcal{H}_{1}$, como no capítulo anterior, devido à 
transformação de variáveis que é feita, devemos apenas atentar para o fato que $Q_{n i}(q)=$ $o\left(\|q\|^{\frac{k}{2}-1}\right)$ e $Q_{(n-1) i}(q)=o\left(\|q\|^{\frac{k}{2}-1}\right) \operatorname{com}\|q\| \rightarrow 0$, para $i=1, \ldots, n$. Desta forma, a extensão dos resultados do capítulo anterior é imediata, e as apresentamos nos corolários abaixo.

Corolário 2. Considere o sistema lagrangeano com forças giroscópias $Q$ dado pelas equações (5.1). Admita ainda que $\mathcal{L}$ satisfaz $\mathcal{H}_{1}$, que $n-N=2$, que $j^{k} \pi_{2}$ na origem não é homogêneo ou é uma sela homogênea e que $Q_{n i}(q)=o\left(\|q\|^{\frac{k}{2}-1}\right)$ e $Q_{(n-1) i}(q)=$ $o\left(\|q\|^{\frac{k}{2}-1}\right)$ com $\|q\| \rightarrow 0$, para $i=1, \ldots, n$. Então existe uma trajetória $\phi(t)$ tal que $(\phi(t), \dot{\phi}(t)) \rightarrow(0,0)$ com $t \rightarrow-\infty$.

Corolário 3. Considere o sistema lagrangeano com forças giroscópias $Q$ dado pelas equações (5.1). Admita $\mathcal{L}=T-\pi$ é tal que 0 é um ponto de equilíbrio e que existe um natural $k \geq 2$ tal que o jato $k$ de $\pi$ mostra que 0 não é um ponto de mínimo para $\pi$, que $\mathcal{L}$ é de classe $\mathcal{C}^{k+3+\left\lfloor\frac{k-3}{2}\right\rfloor}$, que $j^{2} \pi$ na origem é uma forma quadrática semidefinida positiva de posto $n-2$, que $T$ é uma forma quadrática positiva definida em $\dot{q}$ para todo q e que $Q_{n i}(q)=o\left(\|q\|^{\frac{k}{2}-1}\right)$ e $Q_{(n-1) i}(q)=o\left(\|q\|^{\frac{k}{2}-1}\right)$ com $\|q\| \rightarrow 0$, para $i=1, \ldots, n$. Então existe uma trajetória $\phi(t)$ tal que $(\phi(t), \dot{\phi}(t)) \rightarrow(0,0)$ com $t \rightarrow-\infty$. 


\section{Capítulo 6}

\section{Considerações diversas}

Conforme já mencionamos, grande parte da motivação inicial desse trabalho foi a de obter o corolário 1. Entretanto, o teorema 1, que consideramos o principal resultado do texto, facilmente inclui situações diferentes das que foram apresentadas até agora. Dados nossos objetivos iniciais, preferimos pela clareza do texto ignorar essas situações até o momento. Neste capítulo, buscamos então comentar algumas das mudanças tornando as hipóteses um pouco mais fracas que poderiam ter sido feitas sem, entretanto, alterar as demonstrações e técnicas envolvidas e exemplificar as alterações e resultados.

Desta forma, essas observações são pertinentes como possíveis indicações de direções onde se possa procurar resultados melhores que os apresentados em busca de se mostrar a conjectura apresentada na introdução, de que se o jato $k$ da energia potencial mostra que esta não tem mínimo no ponto de equilíbrio, então este é instável.

Manteremos a notação dos capítulos 1, 2 e 3, e pensaremos sempre nas equações de Langrange para o caso conservativo. Os mesmos comentários valem igualmente para os resultados análogos com forças giroscópicas, e por isso não faremos mais menções explícitas sobre esse caso. 


\subsection{U pode ser negativa}

Uma das idéias centrais quando se faz o splitting é de que a parte "estável", isto é, aquela em que a energia potencial é positiva, não vai influir no comportamento da estabilidade. Esta idéia vem a partir do teorema de Dirichlet-Lagrange, imaginando-se que o splitting preserve um pouco o comportamento dos dois sistemas envolvidos: o de energia potencial $U$ e o de energia potencial $\pi_{2}$. Quando a energia cinética é formada por dois blocos, um em $\mathbb{R}^{N}$ e outro em $\mathbb{R}^{n-N}$, que efetivamente separam os sistemas, este pensamento é de fato muito natural. A questão, então, é justamente a influência do acoplamento dos dois sistemas, que ocorre devido aos demais termos da energia cinética. É isto que faz, por exemplo, com que se exijam as condições de acoplamento fraco em Maffei, Moauro e Negrini (1991). Neste trabalho mostramos, entretanto, que pedir que $U$ seja positiva já é uma condição forte o suficiente, e não pedimos qualquer hipótese sobre o acoplamento, exceto quando usamos o resultado de Maffei, Moauro e Negrini (1991) para o caso homogêneo do splitting no plano.

Fica então a questão: se uma parte estável não influi na instabilidade do ponto de equilíbrio, adicionar uma parte instável, ou seja, com energia potencial $U$ negativa (ou mesmo sem mínimo na origem), não deveria dar ainda mais motivos para a instabilidade do ponto de equilíbrio?

Em poucas palavras, dado que de fato não temos resultados nesse sentido além do que comentamos a seguir, a resposta é que numa situação dessas ocorre o mesmo que no caso homogêneo no splitting no plano: o surgimento de mais uma parte instável nos tira o controle de onde está a trajetória assintótica. Assim, não se garante a instabilidade com o método que utilizamos, pois este busca uma trajetória assintótica em uma direção privilegiada - no nosso caso a da n-ésima coordenada - e quando temos duas ou mais direções, não é mais tão claro onde buscar a trajetória assintótica. 
Isso aparece rapidamente quando se segue nossa técnica por, entre outros motivos, tipicamente perdermos as estimativas do lema 1.

Entretanto, isso se deve aos casos em que a $U$ é tão ou mais fortemente negativa que a $\pi_{2}$. Ou seja, quando incluímos uma direção em que $U$ é pelo menos negativa de ordem $\beta$, como a parcela $-\alpha q_{n}^{\beta}$ de $\pi_{2}$. Em vista do que discutimos no parágrafo anterior, isto é justamente consequência de que agora essa direção pode ser a que deveríamos olhar para encontrar uma trajetória assintótica. Para esclarecer melhor esses comentários, vamos discutir agora uma situação exemplo, em que $U$ e $\pi_{2}$ são ambas funções de duas variáveis, então, enunciamos a melhor hipótese sobre $U$ possível nesse contexto.

\subsection{1 $U$ e $\pi_{2}$ como funções de duas variáveis}

Considere um lagrangeano $\mathcal{L}$ que satisfaz $\mathcal{H}_{0}$ a menos da hipótese sobre $U$. Ainda, considere que $N=2$, ou seja, $U$ também é uma função de duas variáveis, e vamos admitir que ela satisfaz hipóteses similares às que $\pi_{2}$ satisfaz. Isto é, suponha que existem reais $\alpha_{0}>0$ e $\beta_{0}>1$ tais que

$$
U\left(q_{1}, q_{2}\right)=-\alpha_{0} q_{2}^{\beta_{0}}+P_{0}\left(q_{1}, q_{2}\right)+R_{0}\left(q_{1}, q_{2}\right),
$$

tal que $P_{0}\left(0, q_{2}\right)=0, P_{0}$ satisfaz a propriedade- $\left(\alpha_{0}, \beta_{0}\right), R_{0}=o\left(\left\|\left(q_{1}, q_{2}\right)\right\|^{\beta_{0}}\right)$ e $\frac{\partial R_{0}}{\partial q_{i}}=$ $o\left(\left\|\left(q_{1}, q_{2}\right)\right\|^{\beta_{0}-1}\right)$ para $i=1,2$. Também, chamando para fins de simplificar a notação de $C^{\prime}$ o análogo a $C$ dado pela propriedade- $\left(\alpha_{0}, \beta_{0}\right)$ de $P_{0}$, temos que exigir que $P_{0} \geq 0$ em $C^{\prime}$.

Com esta notação, desejaríamos fazer a mesma construção que fizemos antes. Notamos, inicialmente, que estamos claramente distantes das hipóteses que fizemos e que nestas condições, sem alteração alguma, estimativas como que $V \geq 0$ nos conjuntos $C_{\sigma_{1}, \lambda}$ não são válidas. E essa é uma etapa vital para que tenhamos a equação (2.2), que 
é uma etapa fundamental na demonstração do teorema 1.

Devemos considerar três casos: $\beta_{0}>\beta, \beta_{0}<\beta$ e $\beta_{0}=\beta$. Com as hipóteses que fizemos, os dois primeiros casos são análogos se trocamos $U$ por $\pi_{2} \mathrm{e}$, desta forma, basta considerarmos os casos $\beta_{0}>\beta$ e $\beta_{0}=\beta$.

Vejamos o caso $\beta_{0}>\beta$. Para isso, a única alteração que temos é que devemos fazer uma pequena alteração na função auxiliar, que agora fica

$$
V(q, \dot{q})=\alpha q_{4}^{\beta}-\frac{1}{2 g_{44}} p_{4}^{2}+\alpha_{0} q_{2}^{\beta_{0}}-R_{0}\left(q_{1}, q_{2}\right)-R\left(q_{3}, q_{4}\right)
$$

e, também, devemos fazer uma pequena modificação em nossa definição de $C_{\sigma_{1}}$, que é pedir que $\left(q_{1}, q_{2}\right) \in C^{\prime}$. Esta última alteração é sutil mas importante, pois ela garante que $P_{0} \geq 0$ no conjunto $C_{\sigma_{1}, \lambda}$, com $\sigma_{1}$ suficientemente pequeno, e assim obtemos da mesma forma a equação (2.2) que gostaríamos. Notamos que, no caso em que $\beta_{0}<\beta$, a diferença na $V$ seria que teríamos que definir $p_{2}(q, \dot{q})=\sum_{s=1}^{2} g_{2 s} \dot{q}_{s}$, ou seja, analogamente ao que fizemos antes, $p_{2}$ é a transformação de Legendre de $\dot{q}_{2}$; e então o termo $\frac{1}{2 g_{44}} p_{4}^{2}$ seria substituído por $\frac{1}{2 g_{22}} p_{2}^{2}$.

Com isso, dado que $\beta_{0}>\beta$ e que $R_{0}$ satisfaz hipóteses similares às de $R$ e, no caso, ainda com ordem de grandeza maior, teremos que os termos nos cálculos dos lemas serão todos trivialmente de ordem superior aos que apareciam. Particularmente, para o cálculo de $\dot{\widetilde{V}}$, todos serão trivialmente $o\left(q_{4}^{\frac{3 \beta}{2}}\right)$, e a demonstração do teorema segue então exatamente como antes, e temos um splitting onde na verdade $U$ só precisa ter $j^{k} U \geq 0$, lembrando que que $k=\lceil\beta\rceil$, e todas as condições necessárias para se demonstrar o teorema neste caso, como discutidas no parágrafo anterior, estarão satisfeitas.

Resta estudar o caso em que $\beta_{0}=\beta$. Este caso é o caso não trivial que ilustra o que chamamos a atenção antes: temos neste caso duas direções, $q_{2}$ e $q_{4}$, que garantem que a 
origem não é um mínimo para a energia potencial com mesma ordem de grandeza $\beta$. E na demonstração dos lemas, logo no lema 1 vamos encontrar dificuldades pois, supondo como antes que continuamos procurando uma trajetória assintótica olhando na direção $q_{4}$ e assim usando a mesma função auxiliar acima, o termo $\alpha_{0} q_{2}^{\beta}$ que aparece neste caso

- lembre que $\beta_{0}=\beta$ - não desaparecerá na limitação superior de $\dot{q}_{4}$ do lema 1 e, pior que isso, será responsável por um termo restante na estimativa do sinal de $\dot{\widetilde{V}}$ que não sabemos comparar diretamente com os restantes nas equações finais deste lema. Desta forma, exemplificamos nesta situação simples o que ocorre ao se acrescentar uma parte que seja de mesma grandeza no sistema.

\subsubsection{As condições sobre $U$}

Com base na nossa discussão e no caso que vimos acima, fica claro que a técnica funciona mesmo quando $U$ tenha regiões negativas, mas o fundamental é que estas não sejam tão "fortes". Por "fortes", entedemos aqui justamente a ordem dos termos que são predominantes para determinar o sinal da função quando estamos suficientemente perto da origem. Assim, se $\beta<\beta_{0}$, podemos pensar que a direção que possui termos de ordem $\beta$ é "mais forte" por "comandar" o sinal da função. Esta, claro, não é uma definição formal, apenas visa tentar esclarecer como podemos ver o problema de uma forma mais natural, pois analiticamente já comentamos que não temos ainda como tratar o problema completamente. O exemplo que apresentamos na verdade é um caso particular mais interessante, onde de fato escolhemos qual a direção de "maior força" que mostra que a energia potencial não tem mínimo na origem e faz a construção em cima desta direção.

Quando se tem hipóteses semelhantes à propriedade- $\left(\alpha_{0}, \beta_{0}\right)$ como na subseção anterior, as demonstrações seguem naturalmente independentemente de $N=2$. Apenas utilizamos este caso por sabermos, conforme o capítulo 4 e que é provado em Garcia 
e Tal (2003), que toda função de duas variáveis cujo jato $k$ mostra que esta não tem mínimo na origem e que exista o jato $(k-1)$ de seu gradiente na origem pode ser escrita de forma a satisfazer as hipóteses apresentadas na subseção.

Desta forma, consolidando a discussão a respeito da $U$, podemos escolher uma das três hipóteses sobre $U$ a seguir, mantendo as demais hipóteses em $\mathcal{H}_{0}$, para obtermos o teorema 1 sem modificarmos sua demonstração exceto por acrescentar na $V$ os termos de ordem superior novos e incluir na definição dos conjuntos $C_{\sigma_{1}}$ que $\left(q_{1}, \ldots, q_{N}\right) \in C^{\prime}$, como fizemos na subseção anterior:

1. $U \geq 0$;

2. $U=j^{k} U+R_{0}\left(q_{1}, \ldots, q_{N}\right)$, sendo $k$ o mesmo da propriedade- $(\alpha, \beta)$ de $P$ em $\mathcal{H}_{0}$, e tal que $j^{k} U \geq 0, R_{0}=o\left(\left\|\left(q_{1}, \ldots, q_{N}\right)\right\|^{k}\right)$ e $\left.\frac{\partial R_{0}}{\partial q_{i}}=o\left(\|\left(q_{1}, \ldots, q_{N}\right)\right) \|^{k-1}\right)$ para $i=1, \ldots, N$;

3. existem reais $\alpha_{0}>0$ e $\beta_{0}>\beta$ tais que

$$
U=-\alpha_{0} q_{N}^{\beta_{0}}+P_{0}\left(q_{1}, \ldots, q_{N}\right)+R_{0}\left(q_{1}, \ldots, q_{N}\right)
$$

onde $P_{0}\left(0, q_{2}\right)=0, P_{0}$ satisfaz a propriedade- $\left(\alpha_{0}, \beta_{0}\right), P_{0} \geq 0$ em $C^{\prime}$ dado pela propriedade- $\left(\alpha_{0}, \beta_{0}\right), R_{0}=o\left(\left\|\left(q_{1}, \ldots, q_{N}\right)\right\|^{\beta_{0}}\right)$ e $\frac{\partial R_{0}}{\partial q_{i}}=o\left(\left\|\left(q_{1}, \ldots, q_{N}\right)\right\|^{\beta_{0}-1}\right)$, para $i=1, \ldots, N$.

Como vimos, no caso $N=2$, o item 3 é um caso particular do item 2 através de uma mudança de coordenadas conveniente. Já no caso $N>2$ isso não é necessariamente verdade. 


\subsection{Alguns exemplos}

Consideramos dois exemplos simples em 3 graus de liberdade que mostram sistemas para os quais os teoremas conhecidos anteriormente ao presente trabalho não garantem a instabilidade da origem. A construção dos exemplos, bem como a aplicação dos nossos resultados e a verificação da não aplicabilidade dos resultados anteriormente presentes na literatura são simples. Mas ainda assim consideramos ilustrativo apresentar os mesmos.

Considere um sistema de energia cinética qualquer e energia potencial dada por

$$
\pi\left(q_{1}, q_{2}, q_{3}\right)=q_{1}^{4}+q_{1}^{2} q_{2}^{4}+q_{2}^{8}-q_{3}^{10}+q_{1} q_{2} q_{3}^{8}+R\left(q_{1}, q_{2}, q_{3}\right)
$$

com $R$ sendo termo de ordem superior e satisfazendo as hipóteses respectivas em $\mathcal{H}_{0}$. Apenas deixamos $R$ aqui para permitir que $\pi$ seja não analítica. Podemos considerar neste exemplo que $\pi=\pi_{2}$ e $N=0$, ou seja, não há $U$. Para esse exemplo, demonstrar a propriedade- $(1,10)$ de $P$ é trivial e temos, assim, que o teorema 1 garante diretamente a existência de uma trajetórtia assintótica para a origem.

Uma pequena modificação do exemplo acima mostra um caso em que temos $U$ com uma região negativa e também uma situação em que pode valer a igualdade na desigualdade que define a propriedade- $(1,10)$ de $P$. Considere

$$
\pi\left(q_{1}, q_{2}, q_{3}, q_{4}, q_{5}\right)=-q_{1}^{11}+q_{2}^{6}+q_{3}^{4}+q_{3}^{2} q_{4}^{4}-q_{5}^{10}+q_{4} q_{5}^{9}+R\left(q_{1}, q_{2}, q_{3}\right),
$$

e temos que $U\left(q_{1}, q_{2}\right)=-q_{1}^{11}+q_{2}^{6}$, que é negativa quando $q_{2}=0$, embora fracamente, como comentamos antes. Observamos aqui que $q_{5} \frac{\partial P}{\partial q_{5}}=9 q_{4} q_{5}^{9}$ e vale a igualdade da propriedade- $(1,10)$ quando $q_{3}=0$ nos pontos da fronteira em que $P=\delta q_{5}^{10}$. 


\section{Capítulo 7}

\section{Conclusão}

Nesta tese avançamos o conhecimento sobre a inversão do teorema de DirichletLagrange e, esperamos, caminhamos positivamente rumo a uma demonstração da conjectura de Liapunov e Barone Netto exposta na introdução.

As técnicas apresentadas, estendendo as de Garcia e Tal (2003), nos permitem uma maior flexibilidade no estudo do problema, mas é importante notar os pontos limitantes das mesmas, que foi o que buscamos fazer no capítulo 6 .

Em particular, destacamos o estudo de $U$ e $\pi_{2}$ como funções de duas variáveis que acreditamos, caso resolvido completamente, possa ser o caminho para uma compreensão mais profunda do problema quando temos mais de uma "direção privilegiada" para buscar trajetórias assintóticas à origem. Em particular, é possível que a compreensão deste caso ajude a entender porque as técnicas que utilizamos não funcionam adequadamente quando o jato $k$ que mostra que não há mínimo na origem para a energia potencial é homogêneo.

Além destes problemas, que estão inseridos na tentativa de demonstrar a conjectura de Liapunov e Barone Netto, resta o difícil problema de se estudar quando o mínimo não 
é mais estrito. Como problema inicial, citamos o caso em que há uma curva de mínimos passando pela origem, onde conhecemos um resultado em 2 graus de liberdade provado por Laloy e Peiffer (1982) em que se mostra a instabilidade da origem, com fortes hipóteses sobre a classe de diferenciabilidade do sistema que, em particular, provam a instabilidade se $\pi$ é analítica e tem um mínimo não isolado na origem.

Desta forma, há ainda várias questões relevantes em aberto na direção do presente trabalho e esperamos que o mesmo contribua no desenvolvimento de técnicas que tornem estes problemas mais acessíveis. 


\section{Referências Bibliográficas}

BARONE NETTO, A. Jet-detectable extrema. Proc. Am. Math. Soc., v. 92, n. 4, p. 604-608, 1984.

BARONE NETTO, A.; GORNI, G.; ZAMPIERI, G. Local extrema of analytic functions. Nonlinear Differential Equations Appl., v. 3, n. 3, p. 287-303, 1996.

FURTA, S. D. On the asymptotic solutions of the equations of motion of mechanical systems. Prikl. Mat. Mekh., v. 50, n. 6, p. 938-944, 1986.

GARCIA, M.; TAL, F. Stability of equilibrium of conservative systems with two degrees of freedom. J. Differential Equations, v. 194, n. 2, p. 364-381, 2003.

LALOY, M. On equilibrium instability for conservative and partially dissipative systems. Int. J. Non-Linear Mechanics, v. 11, p. 295-301, 1976.

LALOY, M.; PEIFFER, K. On the instability of equilibrium when the potential has a nonstrict local minimum. Arch. Rational Mech. Anal., v. 78, n. 3, p. 213-222, 1982.

MAFFEI, C.; MOAURO, V.; NEGRINI, P. On the invertion of the Lagrange-Dirichlet theorem in a case of nonhomogeneous potential. Diff. and Int. Equations, v. 4, n. 4, p. 767-782, 1991.

MOAURO, V.; NEGRINI, P. On the invertion of the Lagrange-Dirichlet theorem. Diff. and Int. Equations, v. 2, n. 4, p. 471-478, 1989. 
PALAMODOV, V. P. Stability of motion and algebraic geometry. In: Dynamical systems in classical mechanics. Providence, RI: Amer. Math. Soc., 1995, (Amer. Math. Soc. Transl. Ser. 2, v. 168). p. 5-20.

ROUCHE, N.; HABETS, P.; LALOY, M. Stability theory by Liapunov's direct method. New York: Springer-Verlag, 1977. xii+396 pp. p. Applied Mathematical Sciences, Vol. 22 .

TALIAFERRO, S. D. Instability of an equilibrium in a potential field. Arch. Rational Mech. Anal., v. 109, n. 2, p. 183-194, 1990. 